\title{
Caracterización del Programa Productivo Alimentario "Hambre Cero". (PPA) en el marco del Plan Nacional de Desarrollo Humano (PNDH) de Estelí, Nicaragua
}

\author{
Elda Dalila Méndez Polanco ${ }^{1}$ \\ Keyling del Carmen Rodríguez Centeno ${ }^{2}$ \\ Naty Jaziel Carrasco Zavala ${ }^{3}$ \\ Katherine Ninoska Pérez Castillo ${ }^{4}$ \\ Beverly Castillo Herrera ${ }^{5}$
}

\section{RESUMEN}

El presente estudio caracteriza el Programa Productivo Alimentario mejor conocido como "Hambre Cero" en la ciudad de Estelí, Nicaragua. El estudio lo realizamos por medio de entrevistas a los coordinadores del Programa a nivel departamental y a sus beneficiarios, también, a través de bibliografías citadas. Este programa surge como la implementación de una política de seguridad alimentaria y nutricional que es justificada en Nicaragua, donde entre 2003 y 2005 un 22\% de la población estaba todavía sub-nutrida, según las cifras de la FAO. Para tal fin el Gobierno de Reconciliación y Unidad Nacional (GRUN) creo el Programa Productivo Alimentario. El objetivo del PPA en sus inicios fue erradicar el hambre, la desnutrición crónica, la pobreza extrema y el desempleo en 75,000 familias rurales pobres, mediante el incremento cuantitativo de la producción y el consumo de alimentos proteicos. Este es uno de los programas más emblemáticos y propagandizados por el actual gobierno como su respuesta principal a las necesidades de las familias campesinas especialmente de las mujeres. Se le presenta como un programa de empoderamiento de las mujeres, ya que la asignación de recursos está dirigida a la mujer de la familia. El programa busca contribuir a reducir la desnutrición y a capitalizar a las familias mediante la comercialización de los productos. En el diseño, es un programa de transferencia en forma de bienes y animales que posteriormente deben reintegrar hasta en un $20 \%$, que será para su propio beneficio, ya que les permite a las mujeres organizarse en cooperativas o emprender su propio negocio. Dentro de los principales resultados están: el $70 \%$ de protagonistas producen y venden sus excedentes, están organizados en 1,681 núcleos solidarios con ellos se han creado 295 cooperativas en las que están integradas 8,111 familias y han generado un fondo revolvente de casi cien millones de córdobas.

Palabras Claves: Hambre Cero; Pobreza; Alimentación; Mujeres; Desarrollo; Nicaragua

Recibido: 17 de noviembre de 2016

Aceptado: 16 de diciembre de 2016

1 UNAN-Managua/FAREM-Estelí. Correo electrónico: yesungjinki@gmail.com

2 UNAN-Managua/FAREM-Estelí. Correo electrónico: keylingrc172@gmail.com

3 UNAN-Managua/FAREM-Estelí. Correo electrónico: carrasconaty22@gmail.com

4 UNAN-Managua/FAREM-Estelí. Correo electrónico: kathypc05@gmail.com

5 Doctora en Ciencias Sociales por la Universidad de Zulia, Venezuela. Maestría en Ciencias Sociales por la Universidad de Guadalajara. Docente titular de la UNAN-Managua, FAREM-Estelí. Correo electrónico: beverly.castillo@yahoo. com 


\title{
Characterization of the Food Production Program "Hambre Cero" (PPA) under the National Plan for Human development of Estelí, Nicaragua
}

\begin{abstract}
The current study characterizes the Food Production Program better known as "Hambre Cero" in Estelí-Nicaragua city. This study was made by the uses of surveys to the program coordinators in the department and also o their beneficiaries but also through bibliography found. This program comes as the implementation of one Nutritional and Food Security politics which is justified in Nicaragua, where between 2003 and 2005 al 22\% (twenty-two percent) of the population were in a sub nutrition way, according to the numbers of FAO. For this purpose the government of Reconciliation and National Unity (GRUN) created the Food Production Program. The purpose of this program on its beginning was to eradicate hunger, the chronic malnutrition, the full poverty and employment in 75,000 (Seventy-five thousand) poor rural families, by quantitative increase in production and consumption of protein foods. This is one of the most emblematic and propagandize program by the current government as it primary answer to the rural family's needs specially women's needs. It is presented as an empowerment program for women, because the resource's destination is guided to the woman. The program aims to contribute, to reduce malnutrition and capitalize to families by marketing products. In the design, it is a program of transfer in the form of goods and animals that then they must repay up until $20 \%$ which will be for its own benefit because it gives to the women to organize them in cooperatives or the undertake their own businesses. Within the main results are: $70 \%$ (seventy percent) of protagonist that produce and sell excedents, they are organized in 1,681 (one thousand, six hundred-ninety five) cooperatives where there are 8,111 (eight thousand, one hundred-eleven) families involved and they have generated a revolving fund almost about one million bucks.
\end{abstract}

Keywords: Hambre Cero, Poverty, Feeding, Women, PPA, Nicaragua. 


\section{INTRODUCCIÓN}

El subdesarrollo económico en América latina no es un hecho reciente. Es un problema viejo, que empieza a gestarse desde hace varios siglos y que se configuran en definitiva cuando los países que se encuentra a la cabeza del mundo capitalista comienzan a convertirse en fuertes naciones industriales y en todo caso lo nuevo es la conciencia cada vez más clara entorno al atraso y sus graves implicaciones, así como la seguridad de que en los países pobres no es imposible aspirar al progreso. Si bien es cierto existe un crecimiento relativo en América Latina, al compararse con otras regiones del mundo y bloques, nuestra región se ubica muy atrás en términos de ingreso per cápita, calidad de vida, seguridad ciudadana, derechos humanos y muchos otros indicadores.

No todos los países en vías de desarrollo están al mismo nivel, lamentablemente algunos se encuentran en posiciones de total desventaja. Los mecanismos para superar este estado son múltiples y variados, y en principio tienen un altísimo costo político, que muchos gobernantes no están dispuestos a asumir, pero en ningún momento debe descuidarse el aspecto humano y social del mismo. Los problemas de desarrollo vienen dados por una serie de características entre las cuales las más comunes están; el sector predominante, la mano de obra barata, los bajos niveles de renta, las organizaciones institucionales no son las más adecuadas, la existencia de fuga de capitales. Todo esto empeora aún más el desarrollo y por lo tanta recaemos en el famoso retraso de desarrollo.

En el caso de la participación ciudadana en América Latina ha tenido mejores resultados debido a que se ha venido dando un fortalecimiento, impulsado por los gobiernos locales y regionales, en donde sus propuestas pretenden posicionar modelos alternativos de desarrollo que logran superar las consecuencias del modelo neoliberal, siendo éstas las que profundizan la exclusión social y la pobreza. La sociedad en América
Latina se caracteriza no por la oposición al Estado ni de manera central por la generación de un mercado en tanto construcción y defensa de la propiedad privada, sino por una combinación de la autoprotección y la idea del movimiento, que a lo largo del último siglo adoptó diferentes significaciones como la ampliación de la participación, revolución, modernización entre otras, no se trata solo de un espacio de construcción de instituciones.

Nicaragua, es el segundo país más pobre de Latinoamérica y el mayor problema para el desarrollo del país es el desempleo. Los ciudadanos enfrentan muchos problemas económicos por la falta de dinero para mantenerse a ellos y a sus familias. Así mismo, recae la falta de educación porque no cuentan con los suficientes recursos para cubrir los gastos escolares para facilitar la educación a sus hijos, por lo tanto, el sufrimiento por el hambre y la falta de educación provoca subdesarrollo al país. Los jóvenes que logran culminar sus estudios universitarios no consiguen empleo por que como requisito se les pide años de experiencia.

El problema de la pobreza se muestra con mayor dureza en el área rural donde el trabajo de los campesinos es la agricultura. Actualmente, el bajo precio de los granos básicos frente a los altos costos que incurren en la siembra no le permite al campesino desarrollarse, en la mayoría de los casos el dinero que obtienen de la venta de la cosecha lo destinan para pagar sus deudas.

Como consecuencia del desempleo y bajos ingresos, los nicaragüenses optan por emigrar a otros países, aspiran a vivir en un país que se inserte exitosamente en el sistema mundial, que les ofrezca oportunidades económicas, sociales y laborales y construye una democracia participativa. Hacer realidad estas aspiraciones demanda la voluntad compartida de la ciudadanía para traducirla en acciones colectivas. 
Nuestra investigación aportara conocimiento a la comunidad universitaria ya que describiremos datos importantes para el estudio de diversas carreras, en ámbitos económicos porque comercializa los excedentes en el mercado nacional e incentivan cadenas de producción agroindustrial, con el objetivo de darle un valor agregado a los bienes producidos o reproducidos en las unidades de reproducción; sociales, porque integra a todas las familias urbanas y rurales nicaragüenses de acuerdo a sus capacidades e integración a mujeres de cualquier rango de edad; y político, porque las políticas desarrolladas en este proyecto están relacionadas con el plan nacional de desarrollo humano, el derecho a la alimentación en Nicaragua e instituciones tales como el Ministerio de Economía Familiar, Comunitaria, Cooperativa y Asociativa (MEFCCA).

En los países latinoamericanos existe bastante pobreza y los gobiernos tratan de combatirla por medio de las políticas públicas. Nicaragua es uno de los países más pobres del continente. En Nicaragua, la lucha contra la pobreza extrema es una necesidad apremiante. Al 2005, el 46 por ciento de los nicaragüenses vivían debajo de la línea de pobreza. Dos de cada tres campesinos nicaragüenses son pobres. El 65 por ciento de los pobres vive en áreas rurales y el 80 por ciento de quienes sufren pobreza extrema, vive en áreas rurales (IEEP, 2009).

Uno de las primeras iniciativas del Nuevo Gobierno de Reconciliación y Unidad Nacional (GRUN) después de su instalación el 10 de enero 2007 ha sido el Programa Productivo Alimentario (PPA), denominado popularmente como "Hambre Cero". El concepto fue derivado de programas similares en otros países latinoamericanos (por ejemplo "Fome Cero" de Brasil), basados en los esfuerzos de crearun colchón de seguridad social y alimentaria a los estratos más empobrecidos del país. El PPA o Hambre Cero es actualmente uno de los programas de la política de soberanía y seguridad alimentaria nicaragüense que conforma -junto con la política de educación, vivienda, salud y abastecimiento de agua y saneamiento- la Política Social del Gobierno de Nicaragua. Fue implementado inicialmente bajo el tutelaje del Ministerio de Agropecuario y Forestal (MAGFOR) que ahora se denomina únicamente como Ministerio Agropecuario (MAG) y en 2013 se le adjudicó al Ministerio de Economía Familiar, Comunitaria, Cooperativa y Asociativa (MEFCCA). El programa tiene coordinación con otras instituciones centrales como el Instituto Nicaragüense de Tecnología Agropecuaria (INTA) y el Instituto Nacional Tecnológico (INATEC).

Como estudiantes de la carrera de Ciencias Políticas, consideramos que es fundamental el estudio y evaluación de los proyectos impulsados por el gobierno de reconciliación y unidad nacional; como es el caso del reto hambre cero, ya que ha sido una iniciativa que tiene como propósito fomentar apoyo para llegar a la meta de eliminar el hambre y fortalecer la producción de alimentos de auto consumo en las familias rurales nicaragüenses. Está investigación brindará información fundamental para todos los estudiantes, dado que abordaremos aspectos relevantes acerca del Programa Productivo Alimentario "Hambre Cero". (PPA) enmarcado en el enfoque de desarrollo y participación ciudadana definido en el Plan Nacional de Desarrollo Humano 2007-2016 de Estelí, Nicaragua.

\section{MATERIALES Y MÉTODOS}

Nuestro objeto de estudio es el Programa Productivo Alimentario (PPA), mejor conocido como "Hambre Cero", es una estrategia implementada por el Gobierno Sandinista para combatir la pobreza en todas sus expresiones.

Nicaragua está situada en el centro del istmo Centroamericano, en la Zona Tropical Norte, entre el Ecuador y el Trópico de Cáncer en el Hemisferio Norte, entre $\operatorname{los} 10^{\circ}$ y $15^{\circ} 45^{\prime}$ Latitud Norte y los $79^{\circ} 30^{\prime}$ longitud oeste. El país tiene una superficie de 
130, $373.47 \mathrm{~km} 2$, correspondiendo 120,339.54 km2 de tierra firme y $10,033.93 \mathrm{~km} 2$ de Lagos y Lagunas (INIDE, 2008). Limita al norte con Honduras, al sur con Costa Rica, al este con el Mar Caribe y al oeste con el océano Pacífico al. Por razones administrativas, Nicaragua se divide en 15 departamentos y dos regiones autónomas. (SIAGUA)

El Programa Productivo Alimentario "Hambre Cero" que se implementa en Nicaragua desde 2007, enmarcado en el compromiso del país por alcanzar la primera meta de los Objetivos de Desarrollo del Milenio de las Naciones Unidas referido a reducir la pobreza extrema y el hambre. El instrumento principal del "Hambre Cero" es el Bono Productivo Alimentario, que consiste en un cierto número de bienes como son: vacas, cerdas, aves, semillas, material vegetal y materiales de construcción hasta un valor total de unos US\$1,500. El Bono se entrega generalmente a la mujer que debe cumplir con ciertas condiciones, por ejemplo la disponibilidad de un patio o una parcela para los animales, así como la disponibilidad de organizarse en "núcleos" y ahorrar/contribuir a un Fondo Revolvente con un monto que equivale al 20\% del valor del Bono. Para los gastos operativos el PPA calcula un monto de US\$ 500 por beneficiaria, llevando el costo total del Programa en los 5 años a unos US\$ 160 millones para 80.000 familias. Los fondos provienen en gran parte del presupuesto nacional, de Alba-Caruna y otros donantes (Kester, 2009)

Esta investigación se desarrolló en tres etapas metodológicas que fueron:

La investigación documental para recaudar la información teórica consultamos sitios web de fuentes confiables, consultamos tesis tanto de graduación como tesis de doctorados; además de visitar la institución encarga del programa como lo es el MEFCCA y entrevistar a los coordinadores y promotores del programa del bono productivo a nivel departamental; además entrevistamos a una secretaria política de la comunidad Los plancitos en el municipio de San Nicolás la cual nos brindó información sobre cómo se lleva a cabo el programa en esa comunidad.

Diseño y aplicación de instrumentos aplicamos entrevistas a los coordinadores del programa a nivel departamental, lamentablemente a los técnicos de dicho programa no les realizamos entrevista debido a la falta de tiempo por parte de los técnicos ya que son muy pocas y deben cubrir varias actividades en diferentes lugares de la ciudad. Viajamos al municipio de San Nicolás a entrevistar a las protagonistas, pero debido a la ausencia de ellas, entrevistamos a la secretaria política de dicho municipio que nos relató cómo se ha llevado a cabo el programa en ese lugar.

Elaboración del informe final el informe final fue elaborado a partir de la guía proporcionada por la docente Dra. Beverly Castillo, que nos permitió tener un orden lógico del trabajo y así brindar una mayor asimilación de la información por parte de los lectores.

\section{RESULTADOS Y DISCUSIÓN}

\section{Tipos de desarrollo en América Latina}

Existen tres tipos de desarrollo que han ganado auge en los últimos tiempos y nos han permitido enfocarnos en el desarrollo teniendo en cuenta la integración humana, la igualdad y la preservación del medio ambiente los cuales son:

Desarrollo con equidad: es, ante todo, una estrategia de desarrollo que implica conjugar, a través de decisiones de autoridad y prácticas colectivas acordes, crecimiento, cambios y reajustes estructurales en la economía, en las relaciones sociales y en el acceso de diferentes sectores de población a recursos, en una perspectiva de mediano y largo plazo. Involucra, por lo tanto, cambios en las relaciones de poder entre actores, en cuanto el ejercicio del poder se hace siempre a través de la movilización de recursos materiales y 
simbólicos, fácticos e institucionales, que se encuentran desigualmente distribuidos (Vilas, 2007).

Desarrollo Sostenible: El IUCN (Instituto Internacional para la Conservación de la Naturaleza) define que el desarrollo sostenible "se basa en el manejo y conservación de los recursos naturales en la orientación del cambio tecnológico e institucional, de tal manera que asegure la continua satisfacción de las necesidades humanas para las generaciones presentes y futuras". (Acosta, 2011)

Desarrollo Humano: La noción de desarrollo humano implica un compromiso con la libertad frente a la opresión política, pero también frente a la opresión de la miseria, la ignorancia y la falta de oportunidades económicas. Al definir como fin último el crecimiento de las capacidades humanas coloca a la gente en el centro de sus preocupaciones, a través de procesos construidos por y desde las personas y las comunidades como verdaderos protagonistas.

\section{El Modelo Cristiano, Socialista y Solidario del Poder Ciudadano en Nicaragua ${ }^{1}$}

El 10 de enero de 2007, no sólo se cambió de gobierno, se inició también un cambio de valores, actitudes, prioridades, estilo de gobernar, relaciones de poder y políticas. El punto inicial y final de estas políticas es el desarrollo del ser humano y las familias nicaragüenses, en sus condiciones históricas, culturales, sociales y de políticas nacionales y regionales del Siglo XXI.

En este proceso se está transformando el modelo de sociedad neoliberal por el Modelo Cristiano, Socialista y Solidario del Poder Ciudadano, el cual se basa en valores que guían la construcción de círculos virtuosos de desarrollo humano, la restitución de derechos y el fortalecimiento de capacidades que han

1 GRUN (2012). Plan Nacional De Desarrollo Humano 2012-2016. Versión preliminar de consulta nacional. Managua, Nicaragua. URL: $\quad$ http://www.pndh.gob.ni/documentos/pndhactualizado/ pndh.pdf permitido ir superando los círculos vicios de pobreza y subdesarrollo y revertir en 2009 el incremento de la pobreza observado entre 2001 y 2005 , a pesar de la peor crisis del capitalismo mundial. Esto fue posible por el poder y la participación del pueblo organizado, y como resultado de esa recuperación de valores, apoyando los programas sociales y de capitalización de las familias productoras que han logrado fortalecer sus capacidades y aumentar su participación social y económica.

El modelo también es socialista al construir el Poder Ciudadano como expresión de organización y movilización del pueblo con plena participación y decisión permanente en la construcción de la democracia directa; es solidario porque las políticas públicas y prácticas solidarias parten de la realidad de las familias nicaragüenses, sus necesidades, sus aspiraciones y su situación actual, así como el impacto de los procesos socioeconómicos y políticas públicas actuales y potenciales sobre las familias nicaragüenses, especialmente las más pobres. También se manifiesta en la responsabilidad social compartida de toda la sociedad para el cuido de la infraestructura económica y social del país, así como para todos los bienes públicos comunes.

El modelo tiene un supuesto de que la única manera de transformar Nicaragua es transformando a las y los nicaragüenses, y los logros 2007-2011 lo demuestran, con más de 500,000 personas en alfabetización y post alfabetización, con la restitución del derecho humano y constitucional a la salud y educación gratuita y universal, con más de 100 mil mujeres campesinas capitalizadas en el campo de Hambre Cero y más de 123 mil mujeres pequeñas empresarias capitalizadas en la ciudad con el Programa Usura Cero, entre otros logros.

El PNDH está sustentado en el Modelo Cristiano, Socialista y Solidario del Poder Ciudadano, que tiene al ser humano como el centro del modelo, rescata para 
el pueblo el rol del Estado para liderar el progreso económico y social. Los avances en la reducción de la pobreza y la desigualdad en un contexto de crecimiento económico, de estabilidad macroeconómica y social, a pesar de la crisis económica y financiera internacional, de las alzas de precios de los combustibles y de alimentos, y de los estragos del cambio climático en el país, son los principales resultados del PNDH 20072011.

Con el PNDH se recuperaron valores al superar los antivalores de la sociedad de consumo individualista, depredadora de la solidaridad humana y de la Madre Tierra. Las personas, mayoritariamente en este plan son jóvenes, madres solteras y mujeres, que se movilizan con el apoyo de los programas sociales en favor de los más pobres o los más desprotegidos, como algunas jornadas de vacunación, de limpieza, la alfabetización y post alfabetización, las campañas de reforestación, se han creado nuevos programas con el fin de satisfacer las necesidades de los ciudadanos tales como Programa Amor y Amor para los más chiquitos, Operación Milagro, construcción y mejoramiento de viviendas de familias con escasos recursos, la construcción de nuevos centros educativos, unidades de salud, centros deportivos, entre otros. (Castillo \& Villarreal, 2011)

El PNDH continúa teniendo en alta prioridad el crecimiento económico con incremento del trabajo y reducción de la pobreza y las desigualdades, con estabilidad macroeconómica, con soberanía, seguridad e integración en armonía con la Madre Tierra en beneficio de las familias nicaragüenses.

Entre 2007-2011, al Gobierno de Reconciliación y Unidad Nacional promovió la alianza estratégica entre Gobierno Nacional, Gobiernos locales y Poder Ciudadano, para el fortalecimiento municipal, fomento del desarrollo local, democracia directa y descentralización.

En el periodo 2012-2016, el GRUN continuara fortaleciendo la gestión pública participativa y consolidando la democracia directa a través de la participación del pueblo organizado en municipios, para desarrollar una planificación territorial que genera políticas de beneficio a las familias, así como la participación de estas en el ejercicio de la auditoria social y la responsabilidad social compartida.

El gobierno está haciendo un esfuerzo para que los ciudadanos se integren en la toma de decisiones, pero el pueblo debe poner de su parte ya que deben interesarse por las políticas que se implementan y no solo esperar a que el gobierno haga todo por ellos.

Nicaragua antes del 2007 poseía una disminución de su desarrollo humano, aunque en este mismo año fue muy bajo comparado con los demás países de Latinoamérica. Hoy Nicaragua avanza satisfactoriamente gracias al PNDH implementado por el Gobierno.

\section{Antecedentes del Programa ProductivoAlimentario. "Hambre Cero"}

El BPA tiene su antecedente inmediato en un programa implementado por diversas ONG en Nicaragua, especialmente por el CIPRES que acompañaron a unas 5,000 familias en diversas zonas del país, sobre todo en la región de occidente y del Río San Juan.

Se señala que el BPA tiene su origen o inspiración en su homónimo brasileño FOME ZERO - iniciado con el gobierno del presidente Luis Ignacio da Silva -, aunque difiere considerablemente en su enfoque integrador y su rol como piedra angular de la política social del gobierno brasileño, que es el eje de coordinación de las acciones de al menos instituciones gubernamentales. $\mathrm{Su}$ homólogo nicaragüense es un programa de una de las cuatro políticas - la de Seguridad Alimentaria - que conforman la Política Social del Gobierno de Nicaragua, implementado por el MAGFOR, con limitada coordinación aún con otras instituciones descentralizadas del sector, como el INTA. Hambre cero fue manejado desde su creación en 2007 hasta 
el 2012 por el Ministerio Agropecuario y Forestal (MAGFOR), pero en 2013 paso al nuevo Ministerio de Economía Familiar, Comunitaria, Cooperativa y Asociativa (MEFCCA).

Los programas sociales para el alivio de la pobreza, se llevan a cabo en los siguientes diez países de América Latina: Argentina, Bolivia, Brasil, Chile, Colombia, Costa Rica, Ecuador, México, Nicaragua y Venezuela. A partir de la experiencia de estos países, se intenta develar las principales orientaciones que se les ha dado a los programas contra la pobreza, con énfasis en sus características particulares y en determinados aspectos de su diseño, tales como la incorporación de nuevos sujetos de atención y el papel actual de los beneficiarios. Así, es posible concluir que en materia de esfuerzos destinados a la superación de la pobreza se avanza gradualmente en tres ámbitos: Constituir una red efectiva de protección social para los grupos vulnerables, "afinando" la definición e identificación de esos grupos y ampliando las dimensiones cubiertas por la red, integrar efectivamente a los grupos pobres a la red de protección social tradicional, a través de intervenciones multisectoriales altamente focalizadas, desarrollar acciones de "segundo piso", entendidas como aquéllas que sirven de nexo entre una base de protección social y las oportunidades de desarrollo de los más desfavorecidos y su incorporación a la vida económica y social de sus países.

\section{Estrategia organizativa del Programa Productivo Alimentario. "Hambre Cero"}

Las estrategias del Hambre Cero conocido como programa productivo alimentario están destinadas a las familias más pobres de la zona rural con el fin de superar los índices de la pobreza mediante la entrega de una Vaca, una cerda, gallinas, alimentos para los animales entre otros. Se propone fortalecer la producción de alimentos de autoconsumo en las familias rurales nicaragüenses. Y en una segunda etapa, comercializar los excedentes en el mercado nacional o internacional, al mismo tiempo que incentivar cadenas de producción agroindustrial (embutidos, por ejemplo) y la creación de cajas de ahorro rurales.

Los objetivos de desarrollo agroindustrial se logran por medio de la organización progresiva desde el seno familiar y se pretende llegar hasta la conformación de unidades mayores de asociatividad y autogestión, y desde la producción primaria hasta la gestión de unidades económicas agroindustriales. (Font, 2010)

El recorrido organizativo del programa tiene una perspectiva de mediano y largo plazo y está pensado para que contribuya al desarrollo económico del país: abastecimiento interno de alimentos, ahorro de divisas, agregación de valor a nuestros productos, incremento de la exportación, entre otros.

Desde el punto de vista organizativo los cuatro momentos son los siguientes: En un primer momento se seleccionan y se organizan las familias para recibir el bono de capitalización. En un segundo momento las familias en número de 50 forman una cooperativa. En un tercer momento, varias cooperativas conforman una unión o central de cooperativas. En un cuarto momento las uniones o centrales conforman federaciones de cooperativas. (Font, 2010)

El programa productivo alimentario (Hambre Cero) brinda la oportunidad de producir alimentos para nutrir y proporciona herramientas para salir adelante con el trabajo de las familias campesinas pobres. (Loaisiga, Alemán, Colindres, \& Aburto, 2010)

En junio del 2007, la Asamblea Nacional de Nicaragua aprobó la Ley de Soberanía y Seguridad Alimentaria y Nutricional (SSAN). La Ley, también conocido por Ley 693, tiene unos puntos imprescindibles para la manera en que el país toma control de la cuestión de alimentos y nutrición. Según la política oficial de Nicaragua, "La Seguridad Alimentaria y Nutricional se refiere al estado de disponibilidad y estabilidad en 
el suministrar de alimentos (culturalmente aceptables), de tal forma que todas las personas, todos los días de manera oportuna gocen del acceso y puedan consumir los mismos en cantidad y calidad libre de contaminantes y tengan acceso a otros servicios (saneamiento, salud y educación) que aseguren el bienestar nutricional y les permita hacer una buena utilización biológica de los alimentos para alcanzar su desarrollo, sin que ello signifique un deterioro del ecosistema. La ley declaró el derecho a la alimentación sin discriminación. Además, los artículos de la ley mandan que la mujer tenga un papel fundamental en la seguridad alimentaria. Esto es clave para el desarrollo de Nicaragua. Con una cultura de machismo y exclusión de mujeres, el involucramiento y empoderamiento de mujeres es necesario para crear cambio sostenible.

Tiene una perspectiva de mediano y largo plazo y está pensado para que contribuya al desarrollo económico del país: abastecimiento interno de alimentos, ahorro de divisas, agregación de valor a nuestros productos e incremento de las exportaciones. (Loaisiga, Alemán, Colindres, \& Aburto, 2010)

Según Loaisiga, Alemán, Colindres, \& Aburto (2010) dentro de las estrategias del PPA tenemos:

- Entrega de bienes y capacitación sobre tópicos que van desde autoestima, higiene, manejo de los animales;

- Asociación para acceder a créditos productivos y capacitación financiera por parte del Fondo de Crédito Rural (FCR) y otras instituciones;

- Planes de negocios derivados de los núcleos organizados. De esta manera, es posible observar que existen otras intervenciones de otras entidades del Estado (INATEC, FCR, INTA, etc.) que complementan el PPA.

\section{Principales resultados del Programa Productivo Alimentario. "Hambre Cero"}

Los principales resultados del programa 'Hambre Cero" publicado por el gobierno en el informe del presidente de la republica al pueblo nicaragüense y a la Asamblea Nacional, expresa que desde el 2007 a la fecha, el programa productivo alimentario ha entregado más de 130,000 bonos productivos a familias protagonistas pobres de las áreas rurales y periurbanas. Se han conformado 1,713 núcleos. A diciembre 2013, estos núcleos manejan ahorros revolventes por un monto de C\$ 96, 169, 981 [aproximadamente U \$ 3, 846,799.24 Dólares]. En 2013, 1,195 mujeres se graduaron como Promotoras Solidarias Agropecuarias, a fin de apoyar tecnológicamente a otras mujeres protagonistas del Programa. En 2013 se conformaron 295 nuevas cooperativas, con lo cual se alcanza 871 cooperativas conformadas desde el año 2007, a partir de los núcleos del BPA. Asimismo, 43 cooperativas de mujeres protagonistas desarrollaron pequeños negocios a través de la Metodología Ruta de Aprendizaje. (Castillo B. , 2016, pág. 13)

Más de un millón de nicaragüenses o 150.000 familias dirigidas por las mujeres fueron incorporados en el exitoso programa "Hambre Cero" o el Bono Productivo Alimentario. (Font, 2010)

Se ha logrado que las mujeres tengan acceso a un mayor nivel de alimentación diaria, garanticen la asistencia escolar de sus hijos, cuenten con un ingreso más estable, obtengan mayores niveles organizativos y participen en el desarrollo de la comunidad ejerciendo un liderazgo horizontal.

Pero el resultado más esperanzador está en las caras de las mujeres. Ningún número, ninguna estadística puede reflejar lo que se detecta cuando vas a su espacio, a su terreno. Están contentas, se las ve comprometidas. Están desarrollando sus capacidades para generar cambios en su realidad concreta. Cambios 
que mejoran su situación y la de los suyos. Cambios que apetece impulsar, conocer y difundir desde y hacia la solidaridad internacional.

Para tener mejores conocimientos sobre lo que es el Hambre Cero en Nicaragua, visitamos a la secretaria política hermana de una beneficiaria del programa en los plancitos San Nicolás-Estelí.

Quintero (2016) afirma que a su hermana el bono se lo entregaron en el año 2008 compuesto de 1 vaca, 10 aves, 1 cerda, 2 bolsa de cemento, 10 metros de malla y 5 láminas de zinc. Desde el año en que recibió el bono, la vaca ha tenido 8 partos, pero debido a que la vaca no daba mucha leche, tuvo que venderla, pero la repuso con una vaquilla que actualmente se encuentra preñada. Y con relación a la cerda la desapareció, pero compró otra esta pare cada 4 meses, las gallinas las cambio por otras, el dinero que obtiene lo ocupa para sufragar los gastos de la economía familiar, dice que su hermana le ha sacado provecho al bono.

Su hermana forma parte de una cooperativa a la cual aportaron un $20 \%$ y estas cooperativas que conforman son comunitarias. Los técnicos las capacitaron a todas las protagonistas para tener mejor conocimiento sobre lo que es cooperativa pero no les dieron todas las horas de capacitación porque no participaron todas las socias. Pero la cooperativa no funciona, dice que está dormida y no han utilizado los fondos, no les han dado funcionamiento.

En las mejoras recomienda más capacitación, saber seleccionar a las beneficiarias, verificar si cuentan con el terreno. Y que la beneficiaria se comprometa a ser amorosa con los animales.

Las demás compañeras de su hermana desaparecieron el bono, no les daban el debido cuido, al contrario, ella si lo conserva a pesar que no tiene terreno, soy yo nos dijo quien le doy espacio en mis tierras para que cuide la vaca y la cerda sin cobrarle alquiler.
Manifiesta que la economía de su hermana ha mejorado porque vende la leche o la cuajada y ese dinero lo ocupa para comprar algunas cosas de la canasta básica como el jabón que antes se le dificultaba comprar, al igual han mejorados sus ingresos económicos.

Según (Rivera, 2016) responsable de Agricultura Familiar en el MEFCA; La estrategia del programa es la restitución de derechos de las familias pobres para mejorar la dieta alimenticia. Las protagonistas son seleccionadas desde la comunidad después se envían al municipio luego al departamento y por últimos a Managua donde son aprobadas para ser parte del bono, pero deben cumplir con los requisitos como lo es tener el terreno suficiente y que esté interesada a capacitarse $\mathrm{y}$ a reproducir el bono, también tienen que tener menos de 10 gallinas y no tener vaca. "Pueden vender los animales, pero tienen que estarlos reproduciendo y reponiendo con otros."

En Estelí para este año fueron entregados 1900 bonos a nivel departamental compuestos por cerdos y aves. El bono vaca se entregó a 34 mujeres las cuales fueron las más exitosas; sin embargo, se tiene propuesto entregar 108 vacas más a las beneficiadas que han tenido éxitos y no se les había podido entregar. Los éxitos no los miden por municipio, pero se dan más en los lugares que no están en el corredor seco.

Actualmente el PPA a cargo del MEFCA entrega solamente las aves y la cerda, aunque cuente con el suficiente espacio para tener la vaca, por ende, la vaca se entrega hasta que miren el cambio o los progresos en las mujeres beneficiadas, el año pasado no se dio vaca. Todo cambio; hasta el 2013 se daba la vaca, pero actualmente se entrega como un estímulo a las mujeres más exitosas. Aunque son pocas las que no han reproducido o no han tenido éxitos con su bono. Los técnicos que vienen de Managua son los que supervisan. 


\section{Participación ciudadana en el Programa Productivo Alimentario. "Hambre Cero"}

El Programa Productivo Alimentario o programa "Hambre Cero" tiene como marco de referencia la democracia política representativa, la democracia política participativa y la democracia directa, lo que implica el respeto a las instituciones, la participación de todas las personas en condiciones de igualdad ciudadana, así como el derecho de la ciudadanía a organizarse para ejercer directamente el poder ciudadano que estipula la constitución. No es pues por casualidad que se haya comenzado a priorizar a las familias campesinas pobres en general y a la mujer en particular.

Sin embargo, la democracia política, que como sabemos no resuelve el problema de la pobreza, necesita completarse a través de la democracia económica, luchando para que todos los sectores marginados acceden a la riqueza nacional, particularmente las mujeres y los pueblos, comunidades indígenas y afrodescendientes del Caribe. Igualmente necesitamos avanzar hacia la democracia cultural que tiene como fin descartar la cultura depredadora de la naturaleza y la cultura de dominio entre las personas, abrazando los nuevos valores de solidaridad, democracia directa y defensa beligerante de los derechos humanos, entre ellos el derecho a la educación y la salud, la vivienda y el empleo. (Font, 2010)

Ante esta situación el Gobierno de Reconciliación y Unidad Nacional se dispone a cambiar las políticas económicas, orientándolas hacia los pequeños y medianos productores empobrecidos, en primer lugar porque son los más necesitados, pero sobre todo por ser los que más producen y porque significan el mayor potencial de desarrollo del país; lo que permitiría cambiar las condiciones de vida de gran parte de la población nicaragüense, del campo y de la ciudad, y posibilitaría el despegue económico que necesita nuestro país.
El Reto del Hambre Cero fomenta la participación de un amplio conjunto de organizaciones, de movimientos sociales y de las personas, alrededor de una visión común. Estas promueven estrategias eficaces, mayores inversiones y más cooperación para el desarrollo, en coherencia conlos acuerdos nacionales e internacionales existentes. Luchan por alcanzar resultados y por rendir cuentas de sus esfuerzos -particularmente a aquellos que sufren de hambre.

Según Kester (2009) se establece que el Programa Productivo Alimentario más conocido como Hambre Cero tendrá un enfoque de género orientado en la propiedad por parte de las mujeres, de los medios de producción, y el trabajo familiar compartido. El abordaje de género será mediante la resolución práctica del nuevo rol de la mujer campesina y el compromiso compartido de los miembros de la familia para el cambio de comportamiento social del hombre en el hogar como escuela de nuevo comportamiento social.

Orientar y capacitar a las mujeres rurales para la buena administración del Bono Productivo es una decisión correcta. Asignarlas bienes de capitalización y promover su organización para lograr mayores impactos en la economía de su hogar y de su comunidad, aumenta su auto-estima como persona y como agente de cambio. Sin embargo, lograr relaciones equitativas de género es un proceso muy largo y requiere un acompañamiento profesional de largo plazo, involucrando además a los hombres.

\section{Sostenibilidad del Programa Productivo Alimentario. "Hambre Cero"}

Cuando surgió el PPA se identificaba como uno de los diversos programas del GRUN orientados a cumplir con el Objetivo de Desarrollo del Milenio que es erradicar la pobreza extrema y el hambre reduciendo a la mitad el porcentaje de personas con ingresos inferiores a un dólar y el porcentaje de personas que padecen hambre. 
Se planteó que dicho programa tendría como instrumento central el Bono Productivo Alimentario (BPA) consistente en un paquete agropecuario con diversos bienes como vaca preñada, cerda cubierta, aves, materiales para las instalaciones y el gallinero, semillas, material vegetativo, plantas frutales y forestales, crédito revolvente, entrenamiento y asistencia técnica en temas como género, salud y alimentación animal, cooperativismo, medio ambiente, comercialización, este paquete tendría un valor máximo de 1,500 dólares incluyendo los gastos operativos y un mínimo de 600 dólares.

Este programa quedó bajo la responsabilidad del MAGFOR e inicialmente beneficiaría a 75,000 familias campesinas empobrecidas en cinco años. En 2009 se vio un gran cambio donde se amplió la meta a 100,000 familias. El bono se entrega generalmente a la mujer que debe cumplir con ciertas condiciones, por ejemplo la disponibilidad de un patio o una parcela para los animales, así como la disponibilidad de organizarse en "núcleos" y ahorrar a un Fondo Revolvente con un monto que equivale al 20\% del valor del Bono.

El programa Hambre Cero inició en el año 2007 con el gobierno de Reconciliación y unidad nacional, desde su inicio el Programa ha contado con diferentes fuentes de financiamientos tanto internos como externos: Tesoro, FONDEAGRO, FIDA, BID, FAO, Austria, China Taiwán y ALBA.

La manera en cómo se han venido creando más capacidades en los grupos de beneficiadas para que paulatinamente se vinculen a la economía local y nacional ha sido de mucha ayuda. El trabajo de los técnicos del PPA en diferentes zonas, ha obtenido un gran logro ya que tienen mucha disponibilidad de atender a las beneficiadas, trabajando y caminando muchas más horas de las establecidas.

Con este programa también se seguirá combatiendo la injusticia social imperante en este país desde la época colonial, la que se expresa en un conjunto de relaciones de desigualdad, entre ellas: división entre el hombre y la mujer, división entre la ciudad y el campo, división entre el trabajo intelectual y el trabajo manual, división entre poseedores y desposeídos, división entre diferentes etnias o naciones (blancos, mestizos, pueblos indígenas, afrodescendientes).

Cuando inició la entrega del Bono Productivo hubo muchas quejas por parte de las beneficiadas, con frecuencia manifestaban que no se les entregaba el bono completo o que la condición de la vaca o de la cerda no era la prometida. Muchas no venían preñadas. De igual manera, relataban que el alimento que les entregaban para los animales sólo les dio para uno o dos meses y después les había tocado asumirlo a ellas, lo que se les dificultaba por su condición de pobreza. Se debe de reconocer que con el tiempo se vino trabajando con las quejas que hacían las beneficiadas, la calidad de los animales ha mejorado y ahora son ellas quienes eligen los animales que van a criar.

Nicaragua y las familias campesinas han incrementado su producción de carne y leche; está comercializando exportando más carne y leche. La pobreza y la extrema pobreza ha disminuido, pero sobre todo la desnutrición infantil. Las cooperativas de las mujeres han entrado en la etapa de gestionar sus fondos evolventes, centros de acopio, procesadoras agroindustriales, centros de exportación de sus productos procesados y empacados.

Gracias a este programa las mujeres hoy en día trabajan para ellas mismas, con la venta de excedentes y de los animales que se producen, algunas de las beneficiarias han hecho mejoras en sus casas, algunas han utilizado el dinero para pagar el dinero. Antes trabajaban de jornaleras, de cocineras o salían a cortar café en las haciendas. Todos trabajos muy pesados realizados en condiciones de explotación y precariedad y donde sus salarios eran bajos. Ahora trabajan para ellas mismas, no para los demás. Es claro que esto es un beneficio para las mujeres de nuestro país, aunque tiene un doble filo para algunas que antes salían a trabajar el cambio a su puesto ha vuelto al espacio tradicional de la casa, en el 
que se le pude llamar a ellas como mujeres productoras e independientes o mujeres emprendedoras.

\section{CONCLUSIONES}

En definitiva el subdesarrollo económico en América latina no es un hecho reciente. Es un problema viejo, que empieza a gestarse desde hace varios siglos y que se configuran en definitiva cuando los países que se encuentra a la cabeza del mundo capitalista, comienzan a convertirse en fuertes naciones industriales y en todo caso, lo nuevo es la conciencia cada vez más clara entorno al atraso y sus graves implicaciones, así como la seguridad de que en los países pobres no es imposible aspirar al progreso.

Los gobiernos latinoamericanos deben trabajar en la integración de los diversos grupos sociales para poder lograr un buen desarrollo y en Nicaragua se está trabajando en pro del desarrollo humano, social y cultural. El Programa Productivo Alimentario (PPA) ha venido a contribuir al desarrollo del país desde el enfoque de género, al ser su objetivo principal el empoderamiento de la mujer campesina en la economía de su hogar.

Como se ha descrito el Programa Alimentario "Hambre Cero" para que tenga éxito, requiere de compromiso y dedicación de las protagonistas y su familia. El manejo del bono productivo representa una forma de autoempleo para las mujeres puesto que tienen que invertir tiempo para alimentar a los animales, recoger los productos y elaborar los subproductos.

Muchas regiones de nuestro país que sufrían hambrunas están ahora en condiciones de satisfacer las necesidades nutricionales de los más vulnerables. Estos son logros significativos para el cumplimiento de las metas establecidas por los Objetivos del Programa Hambre Cero, a pesar de que se ha trabajado mucho en lo que es el hambre y la desnutrición aún queda mucho por trabajar. Hambre cero es uno de los 17 Objetivos Globales de la nueva Agenda para el Desarrollo Sostenible.

\section{BIBLIOGRAFÍA}

Acosta, E. (2011). Desarrollo Sostenible o Sustentable. Obtenido de Desarrollo Sostenible o Sustentable: http://desarrollosostenibleysustentable.blogspot. com/

Castillo, B. (2016). Modelo de abordaje de la identidad de genero en programas sociales, como aporte al feminismo dialogico. Managua, Nicaragua.

Castillo, B., \& Villarreal, M. (2011). Dinámica del Programa Productivo Alimentario "Hambre Cero" de Nicaragua. ORBIS. Revista Cientifica Ciencias Humanas, 11(31), 96-120. Obtenido de: http:// www.redalyc.org/articulo.oa?id=70946592006

Font, E. (2010). El Programa Productivo Alimentario en Nicaragua y su expresión concreta, el Bono Productivo Alimentario. Obtenido de http://www. rebelion.org/noticia.php?id=118766

GRUN. (2012). Plan Nacional de Desarrollo Humano 2012-2016. Versión preliminar de consulta nacional. Managua, Nicaragua. Obtenido de http:// www.pndh.gob.ni/documentos/pndhactualizado/ pndh.pdf

IEEP. (30 de Marzo de 2009). Voces del Hambre Cero. Managua, Nicaragua: IEEP- Instituto de Estudios Estrategicos y Políticas Públicas. Obtenido de Actualidad: http://simas.org.ni/media/voces hambre_cero.pdf

Kester, P. (2009). Informe evalualitivo (2007-2008) Programa Procuctivo Alimentario (PPA) "Hambre cero”. Managua: Koninkrijk.

Loaisiga, J., Alemán, L., Colindres, L., \& Aburto, V. (2010). Los rostros del Hambre Cero. Libre Pensamiento. Obtenido de: http:// librepenicmoncjose.blogspot.com/2010/06/losrostros-del-hambre-cero-una-mirada_19.html

Quintero, M. (13 de Noviembre de 2016). Hambre Cero. (K. Rodriguez, Entrevistador)

Rivera, H. (6 de Noviembre de 2016). Hambre Cero. (E. M. Polanco, Entrevistador)

Sistema Iberoamericano de información sobre el agua. (s.f.). Obtenido de http://www.siagua.org/pais/ nicaragua 